\title{
ANALYSIS OF PHASE-WAVES IN COUPLED OSCILLATORS AS A LADDER
}

\author{
Masayuki YAMAUCHI, Yoshifumi NISHIO and Akio USHIDA \\ Dept. of Electrical and Electronic Engineering, Tokushima University, Japan \\ e-mail \{masa,nishio,ushida\}@ee.tokushima-u.ac.jp
}

\begin{abstract}
In this study, wave propagation phenomena of phase differences observed in van der Pol oscillators coupled by inductors as a ladder are investigated. It is called "phase-wave." We observe that the behavior of the phase-waves generated by giving phase difference of plus value is different from those generated by giving phase difference of minus value. We analyze the difference in detail. Further,we analyze the difference between generated phase-waves by plus and minus value.
\end{abstract}

\section{INTRODUCTION}

Large number of coupled limit-cycle oscillators are useful as models for a wide variety of systems in natural fields, for example, diverse physiological organs including gastrointestinal tracts and axial fiber of nervous systems, convecting fluids, arrays of Josephson junctions and so on. Hence, it is very important to analyze synchronization and the related phenomena observed in coupled oscillators in order to the clarify mechanisms of the generations or in order to control the generating-conditions of various phenomena in such natural systems. In the field of the electrical engineering, a lot of studies on synchronization phenomena of coupled van der Pol oscillators have been carried out up to now.

Recently, we have discovered very interesting wave propagation phenomena of phase states between two adjacent oscillators in an array of van der Pol oscillators coupled by inductors [1]-[3]. In the study, we named the continuously existing wave of changing phase states between two adjacent oscillators from in-phase to anti-phase or from antiphase to in-phase as "phase-inversion-wave" and explained their generation mechanisms by using the relations between the oscillation instantaneous frequencies and the phase differences of two adjacent oscillators.

We observed "phase-wave," which is propagation of the phase difference between two adjacent oscillators [4]. The phase-waves exist only in the transient states unlike the phase-inversion-waves. But, these study only observed the phase-waves by inputting phase difference between the first oscillator and the other oscillators of plus value. In this study, we investigate the phase-waves in more detail again. In the computer calculations, we produce phase-waves as follows. Almost same initial conditions are given for all oscillators to produce complete in-phase synchronization, which is one stable steady states in the system. After the system settles in the complete in-phase synchronization, the voltage and the current of arbitrary phase difference is input in the first oscillator. We observe that the behavior of the phase-waves generated by giving phase difference of plus value is different from that generated by giving phase difference of minus value. We analyze the difference in detail. Further, we analyze the reason of the differences between observed phenomena by giving phase difference of plus and minus value.

\section{CIRCUIT MODEL}

The circuit model used in this study is shown in Fig. 1. N van der Pol oscillators are coupled by coupling inductors $L_{0}$. We carried out computer calculations for the cases of $N=17$. Although we introduce the results only for 17 oscillators, the similar phenomena are observed from both of even and odd number oscillators. In the computer calculations, we assume the $v-i$ characteristics of the nonlinear negative resistors in the each circuit as the following function.

$$
i_{T}\left(v_{k}\right)=-g_{1} v_{k}+g_{3} v_{k}^{3} \quad\left(g_{1}, g_{3}>0\right)
$$

The circuit equations governing the circuit in Fig. 1 are written as

[First Oscillator]

$$
\begin{aligned}
& \dot{x}_{1}=y_{1} \\
& \dot{y}_{1}=-x_{1}+\alpha\left(x_{2}-x_{1}\right)+\varepsilon\left(y_{1}-\frac{1}{3} y_{1}^{3}\right)
\end{aligned}
$$

[Middle Oscillators]

$$
\begin{aligned}
& \dot{x}_{k}=y_{k} \\
& \dot{y}_{k}=-x_{k}+\alpha\left(x_{k+1}-2 x_{k}+x_{k-1}\right)+\varepsilon\left(y_{k}-\frac{1}{3} y_{k}^{3}\right) \\
& (k=2 \sim N-1) \\
& \text { Oscillator] } \\
& \dot{x}_{N}=y_{N} \\
& \dot{y}_{N}=-x_{N}+\alpha\left(x_{N-1}-x_{N}\right)+\varepsilon\left(y_{N}-\frac{1}{3} y_{N}^{3}\right)
\end{aligned}
$$$$
\text { [Last Oscillator] }
$$ 

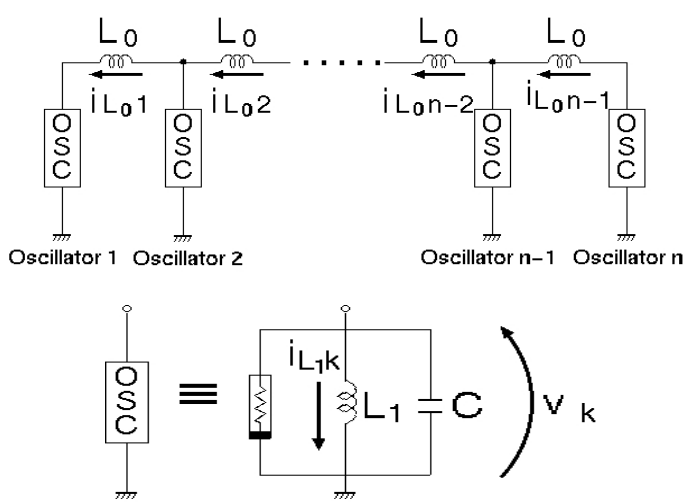

Figure 1: Coupled van der Pol oscillators as a ladder.

where

$$
\begin{aligned}
& t=\sqrt{L_{1} C} \tau, i_{L_{1} k}=\sqrt{\frac{C g_{1}}{3 L_{1} g_{3}}} x_{k}, v_{k}=\sqrt{\frac{g_{1}}{3 g_{3}}} y_{k}, \\
& \alpha=\frac{L_{1}}{L_{0}}, \varepsilon=g_{1} \sqrt{\frac{L_{1}}{C}}, \frac{d}{d \tau}=" . " .
\end{aligned}
$$

It should be noted that $\alpha$ corresponds to the coupling of the oscillators and $\varepsilon$ corresponds to the nonlinearity of the oscillators. In this study, we calculate (2)-(4) by using the fourth-order Runge-Kutta method.

\section{PHASE-WAVES}

\subsection{Phase difference}

In this section, wave propagation phenomenon observed from the circuit with 17 oscillators is investigated.

Throughout the paper, we fix $\alpha=0.050$ and $\varepsilon=0.30$ and we define the phase difference between two adjacent oscillators as follows:

$$
\Phi_{k, k+1}(n)=\frac{\tau_{k}(n)-\tau_{k+1}(n)}{\tau_{k}(n)-\tau_{k}(n-1)} \times \pi
$$

where $\tau_{k}(n)$ is time when the voltage of OSC $k$ crosses $0[\mathrm{~V}]$ at $n$-th time.

Figure 2 shows typical examples of observed phase-waves which are propagation of the phase-difference between adjacent oscillators. These results are obtained for the same parameter values by changing initial conditions as follows:

1. Setting the initial conditions of all oscillators as the same.

2. Putting the arbitrary phase difference of the voltage and the current to one oscillator.

In the upper diagrams of Fig. 2, the vertical axis is the sum of the voltages of adjacent oscillators, and the horizontal axis is time. Hence, the diagrams show how the phase differences between adjacent oscillators change as time goes. In the lower figures, the snapshots of the attractor of each oscillator and the phase states between adjacent oscillators are shown.

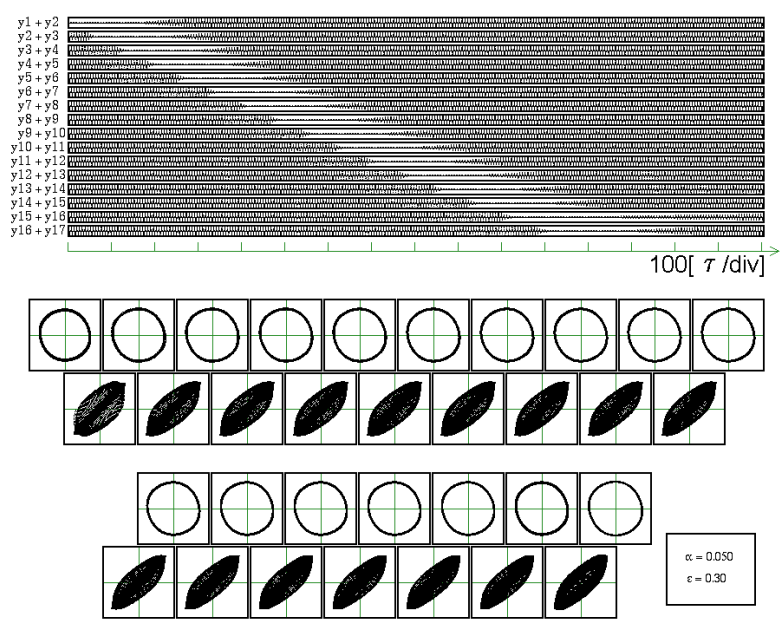

(i) Input phase difference $+60[\operatorname{deg}]($ domain $(\mathrm{a}))$.

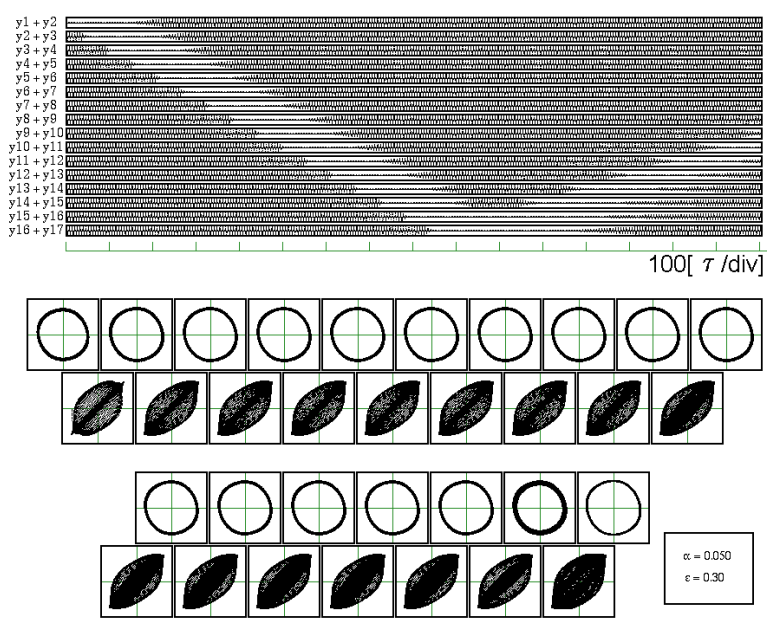

(ii) Input phase difference $+70[\mathrm{deg}]($ domain (b)).

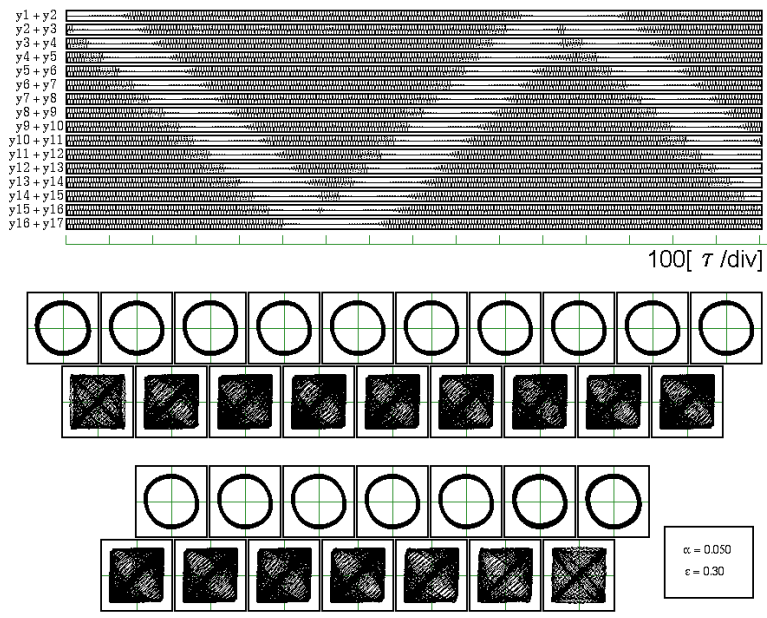

(iii) Input phase difference $+135[\mathrm{deg}]$ (domain (c)).

Figure 2: Phase-waves. $\alpha=0.05, \varepsilon=0.3$. 


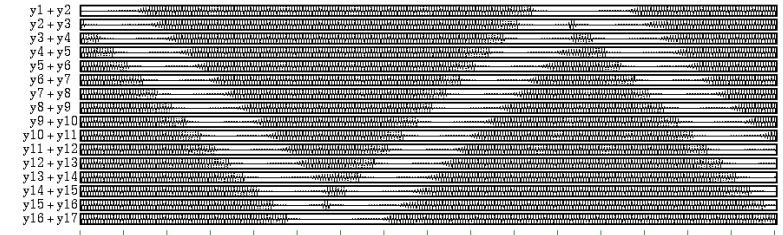

$100\left[\frac{1}{\tau / \mathrm{div}}\right]$

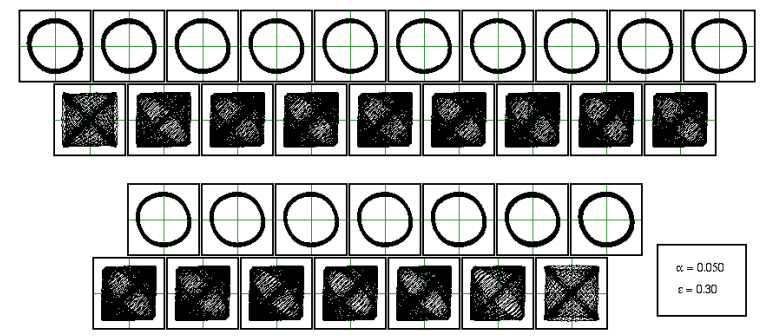

(iv) input phase difference $+175[\mathrm{deg}]$ (domain (d)).

Figure 2: (continued).

\subsection{Classification}

When the phase-waves are generated by inputting the phase difference of plus value or minus value at an edge of the ladder, observed phenomena are classified into four patterns by the input phase difference as follows:

(a) Between $0^{\circ}$ and $+69^{\circ}$ and between $0^{\circ}$ and $-67^{\circ}$ : The phase difference decreases while the phase-waves are propagating. The phase-waves become extinct (1) at the edge of the array without reflection or (2) in the middle of the array ahter one reflection(see Fig.2(i)).

(b) Around $+70^{\circ}$ : The phase difference increases while the phase-waves are propagating. After the phasewaves reflect at the end of the array, they become extinct at the first oscillator. Because the phase-waves do not have enough phase differences to become antiphase at the edge of the array (see Fig.2(ii)).

(c) Between $+71^{\circ}$ and $+171^{\circ}$ and around $-68^{\circ}$ : The phase difference increases while the phase-waves are propagating. The phase-waves reflect at the edge of the array and change to the phase-inversion-waves, because the phase-waves have the phase differences to become anti-phase at the edge of the array (see Fig.2(iii)).

(d) Between $+172^{\circ}$ and $+180^{\circ}$ and between $-69^{\circ}$ and $-180^{\circ}$ : The phase-waves change to the phase-inversionwaves while the phase-waves are propagating (see Fig.2(iv)).

Figure 3 shows domain of the observed phenomena.

Furthermore, we found that two pairs of waves are generated in the domain marked with diagonal line of Fig.3.
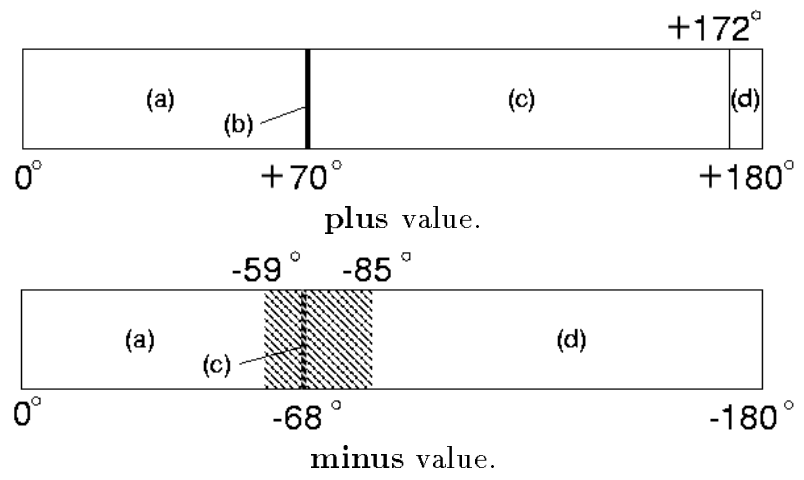

Figure 3: Domain of observed phenomena that are gerated by the phase difference of plus or minus value.

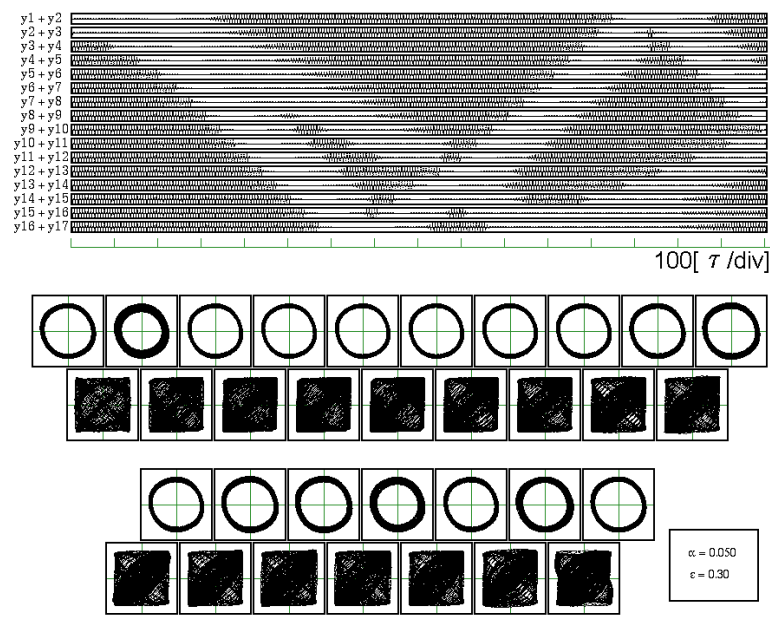

Figure 4: Input phase difference $-70[\mathrm{deg}]$ (domain marked with diagonal line of Fig.3).

Figure 4 shows an example of generated two pairs of waves. This complicated phenomena will be discussed in future.

\subsection{Mechanism of propagation}

Let us assume that all oscillators are in in-phase synchronization. The phase difference of arbitrary plus value inputs into the first oscillator.

(1) Therefore, instantaneous frequencies of the first and the second oscillators $\left(f_{1}\right.$ and $f_{2}$ ) become high frequency from frequency of in-phase synchronization $\left(f_{i n}\right)$. (The frequency of anti-phase synchronization is higher than frequency of in-phase synchronization.) 


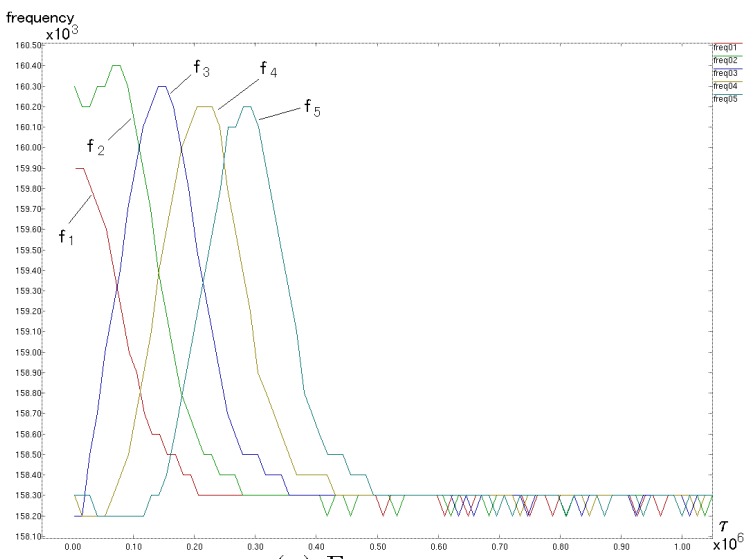

(a) Frequency.

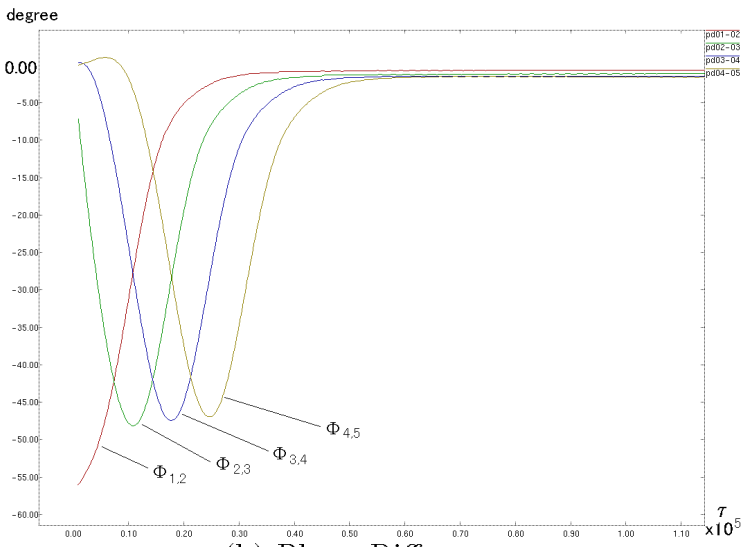

(b) Phase-Difference.

Figure 5: Mechanism of propagation of phase-waves by phase difference of plus value $\left(+60^{\circ}\right)$.

(2) The phase difference between the second and the third oscillators $\left(\Phi_{2,3}\right)$ changes from 0 toward $-\pi$, because $f_{2}$ is higher than $f_{3}$.

(3) $f_{3}$ changes from $f_{i n}$ toward frequency of anti-phase synchronization $\left(f_{\text {anti }}\right)$.

(4) $f_{1}$ becomes $f_{i n}$, because the $\phi_{1,2}$ is small value for becoming instantaneous anti-phase synchronization between the first and the second oscillator.

(5) Therefore, $f_{1}$ and $f_{2}$ change toward $f_{i n}$.

(6) The $\Phi_{2,3}$ changes toward 0 , because $f_{2}$ is smaller than $f_{3}$.

(7) $f_{3}$ becomes $f_{i n}$.

When value of propagating phase difference is in the region attracting to anti-phase synchronization, the phasewaves change toward the phase-inversion-waves. When value of propagating phase difference is not in the region attracting to anti-phase synchronization, the phase-waves change toward extinction.
3.4. Cause of the differences between phenomena by phase difference of plus value and minus value

We observed that the behavior of the phase-waves generated by giving phase difference of plus value is different from that generated by giving phase difference of minus value. We analyze the difference in detail.

When the phase-waves are generated by minus value, we can observe the phenomena same as those by the plus value in the domain between $0^{\circ}$ and $-58^{\circ}$. But, we can not observe the phenomena same as those by the plus value in the domain between $-59^{\circ}$ and $-180^{\circ}$.

When the phase-waves are generated by the phase difference of $+70^{\circ}$, the phase of the second oscillator has to change $70^{\circ}$ to catch up with the phase of first oscillator. While, when the phase-waves are generated by the phase difference of $-70^{\circ}$, the phase of the second oscillator has to change $290^{\circ}$ to catch up with the phase of first oscillator. Therefore, it takes long time that the first and the second oscillator become in-phase synchronization.

\section{CONCLUSION}

In this study, the phase-waves were investigated by numerical calculation. We found that the behavior of the phasewaves generated by giving phase difference of plus value is different from that generated by giving phase difference of minus value. We analyze the difference in detail. When the phase-waves are generated by giving phase difference of minus value, we found the phenomena that phase-waves of two sets generate. Further, we analyzed the differences between generated the phase-waves by the phase difference of plus and minus value.

\section{REFERENCES}

[1] M.Yamauchi, M.Wada, Y.Nishio and A.Ushida, "Wave Propagation Phenomena of Phase States in Oscillators Coupled by Inductors as a Ladder," IEICE Trans. Fundamentals, vol.E82-A, no.11, pp.2592-2598, Nov. 1999.

[2] M.Yamauchi, M.Wada, Y.Nishio and A.Ushida, "Collisions between Two Phase-Inversion-Waves in an Array of Oscillators,"

Proc. ISCAS'O0, vol.1, pp.679-682, May 2000.

[3] M.Yamauchi, Y.Nishio and A.Ushida, "Reflection and Transmission of Phase-Inversion-Waves in Oscillators Coupled by Two Kinds Inductors," Proc. ISCAS'01, vol.3, pp.767-770, May 2001.

[4] M.Yamauchi, M.Wada, Y.Nishio and A.Ushida, "Analysis of Phase Difference Propagation in Oscillators Coupled by Inductors as a Ladder," Proc. NOLTA'99, vol.2, pp.783-786, Nov. 1999. 